\title{
Estilos educativos paternos y estrategias de aprendizaje en alumnos de Educación Secundaria
}

\author{
$\mathrm{M}^{\mathrm{a}}$ Teresa Cerezo, Pedro F. Casanova, Manuel J. de la Torre y \\ $\mathrm{M}^{\mathrm{a}}$ de la Villa Carpio \\ Universidad de Jaén (España)
}

\begin{abstract}
El objetivo del presente estudio consistió en determinar cómo los estilos educativos paternos inciden sobre la utilización de estrategias de aprendizaje autorregulado de un grupo de estudiantes de Educación Secundaria y cómo dichas estrategias, a su vez, influyen sobre el rendimiento académico. Cuatrocientos setenta y ocho estudiantes de educación secundaria obligatoria, con edades comprendidas entre los 12 y los 16 años, formaron parte de esta investigación. Para la evaluación de los estilos educativos paternos se elaboró una escala y la medición de las estrategias de aprendizaje autorregulado se llevó a cabo mediante el Inventario de Estrategias de aprendizaje y Estudio LASSI. Además se tuvieron en cuenta las calificaciones obtenidas por los estudiantes en tres asignaturas en el curso anterior. Los resultados obtenidos muestran cómo aquellos alumnos cuyos padres son clasificados como democráticos y como permisivos utilizan en mayor medida estrategias de aprendizaje autorregulado si los comparamos con los estudiantes cuyos padres son clasificados como autoritarios y negligentes. Además, se ha podido constatar cómo este aprendizaje autorregulado incide positivamente sobre el logro académico de los alumnos.
\end{abstract}

Palabras clave: Adolescentes, estilos educativos paternos, estrategias de aprendizaje.

Parents' educational styles and self-regulated learning strategies in a group of Secondary Education students. The purpose of the present study was to state how parents' educational styles affect over the use of self-regulated learning strategies in a group of Secondary Education students and how mentioned strategies also influence on their academic performance. Four hundred and seventy eight Secondary Education students with ages between 12 and 16 took part in this investigation. In order to evaluate parents' educational styles a set of values was made based on the instrument and the measuring of the self-regulated learning strategies was done through the Inventory of Learning Strategies and Study LASSI. Furthermore, students' marks in three different subjects during the previous year were taken into consideration. The obtained results showed how those students whose parents are classified as democratic and permissive more often use self-regulated learning strategies when compared to children of authoritarian and negligent parents. Besides, it has been stated how this self-regulated learning positively influence over academic successful results.

Key words: Adolescents, parenting styles, learning strategies.

Correspondencia: $\mathrm{M}^{\mathrm{a}}$ Teresa Cerezo. Departamento de Psicología. Universidad de Jaén. Paraje de las Lagunillas S/n. C.P. 23071. Jaén (España). E-mail: macerezo@ ujaen.es 
Durante las dos últimas décadas han sido frecuentes los trabajos realizados sobre los estilos educativos paternos y su influencia sobre el aprendizaje y logro académico de los estudiantes (Ballantine, 2001; Balzano, 2002; Burchinal, PeisnerFeinberg, Pianta y Howes, 2002; Glasgow, Dornbusch, Troyer, Steinberg y Ritter, 1997; Peralbo y Fernández, 2003; Pérez De Pablos, 2003). Dichos estilos han sido definidos como esquemas prácticos de conducta que reducen las múltiples pautas educativas paternas a unas pocas dimensiones básicas que, cruzadas entre sí, dan lugar a diversos tipos habituales de educación familiar (Coloma, 1993).

Diversas clasificaciones identifican una serie de estilos educativos paternos, mediante la combinación de las dimensiones de afecto y control de los padres hacia los hijos. Los padres autoritarios, presentan alto nivel de control y bajo nivel de afecto, consideran la obediencia como una virtud, no suelen dialogar con sus hijos y favorecen las medidas de castigo, restringiendo la autonomía, iniciativa y espontaneidad del hijo (Baumrind, 1991; Lamborn, Mounts, Steinberg y Dornbusch, 1991; Maccoby y Martin, 1983).

Los padres democráticos, caracterizados por altos niveles de control y de afecto, estimulan la comunicación bidireccional y establecen normas que regulan su conducta. Según Torío, Peña y Rodríguez (2008), estos padres otorgan gran importancia a la iniciativa personal del niño, aceptan los errores que los hijos puedan cometer y fomentan el respeto y reconocimiento mutuo.

Los padres permisivos, con bajo control y alto nivel de afecto, son tolerantes y afectuosos, evitan las restricciones y castigos, plantean pocas exigencias a sus hijos y les permiten una considerable autorregulación. Por último, los padres indiferentes, que muestran bajos niveles de control y de afecto, se caracterizarían por no involucrarse afectivamente en los asuntos de los hijos y por la falta de implicación en la tarea educativa. Les resulta más cómodo no poner normas, pues éstas implicarían diálogo y vigilancia, tendiendo a resolver las obligaciones educativas de la manera más rápida y cómoda posible (Torío, Peña e Inda, 2008).

Trabajos recientes han puesto de relieve la relación entre estos estilos educativos y el rendimiento académico obtenido por los hijos (Glasgow et al., 1997; Hickman, Bartholomae y McKenry, 2000; Pelegrina, García y Casanova, 2002; Peralbo y Fernández, 2003; Pérez De Pablos, 2003). Sin embargo, dicha relación es indirecta, ya que los estilos y prácticas educativas paternas inciden significativamente sobre las características cognitivas y motivacionales implicadas en el aprendizaje escolar, relacionándose éstas a su vez, con los logros académicos obtenidos por los estudiantes. Por ejemplo, en diversos estudios se ha constatado que los estilos y las prácticas educativas paternas están relacionados con variables motivacionales y con la competencia percibida. Así, se ha relacionado el estilo educativo democrático con el desarrollo de una orientación motivacional intrínseca y los estilos autoritario y permisivo 
con una orientación motivacional extrínseca (Dornbusch, Ritter, Leiderman, Roberts y Fraleigh, 1987; González, Holbein y Quilter, 2002; Grolnick y Ryan, 1989; Steinberg, Elmen y Mounts, 1989). Además, existe evidencia de que los estilos educativos paternos inciden también sobre la competencia académica percibida (González-Pienda et al., 2002; Pelegrina et al., 2002), de tal manera que los hijos que evalúan a sus padres como democráticos o permisivos se consideran a sí mismos más competentes que los hijos de padres autoritarios o indiferentes.

\section{Estrategias de aprendizaje autorregulado}

Son abundantes los trabajos que abordan el estudio de la influencia de los padres sobre la motivación, el autoconcepto, el esfuerzo, etc. de sus hijos, asumiendo que tales variables sensibilizan al estudiante hacia la utilización de procesos y estrategias cognitivas, las cuales, incidirán significativamente sobre el aprendizaje y el rendimiento. Sin embargo, son escasas las investigaciones que analizan cómo las prácticas educativas paternas pueden favorecer o dificultar el aprendizaje autorregulado, entendiendo éste como una serie de procesos mediante los cuales los estudiantes activan cogniciones, afectos y conductas que están sistemáticamente orientadas hacia la consecución de las metas de aprendizaje (Schunk y Zimmerman, 2008).

Uno de los componentes del aprendizaje autorregulado son las estrategias de aprendizaje autorregulado, las cuales incluyen estrategias cognitivas y metacognitivas de aprendizaje (Pintrich, 1999). Las estrategias cognitivas se refieren a los distintos procesos y conductas que los estudiantes ponen en marcha durante la realización de las tareas académicas con el fin de conseguir las metas de aprendizaje. Las estrategias metacognitivas, sin embargo, se refieren al conocimiento y el control que el estudiante tiene de los propios procesos psicológicos implicados en el aprendizaje.

Otro de los componentes del aprendizaje autorregulado son las creencias motivacionales. Pintrich (1999) estableció cuatro tipos de creencias motivacionales: creencias de autoeficacia, creencias sobre el valor de la tarea, orientación hacia las metas y ansiedad ante la evaluación.

Teniendo en cuenta que el cambio de perspectiva sobre el aprendizaje escolar, desde el clásico modelo cognitivo al modelo de aprendizaje autorregulado, ha supuesto también una nueva orientación para la investigación acerca de la influencia de la familia sobre el estudio y aprendizaje escolar de los hijos, en este trabajo se pretende estudiar cómo los estilos educativos paternos inciden en la utilización de estrategias de aprendizaje autorregulado de un grupo de estudiantes de Educación Secundaria y cómo estas estrategias influyen sobre el rendimiento académico. En concreto se espera encontrar diferencias significativas en el uso de estas estrategias en función del estilo educativo paterno, siendo el estilo democrático el más beneficioso en este sentido. También se espera encontrar diferencias significativas en el rendimiento académico en 
función del uso de estrategias de aprendizaje autorregulado, de tal manera que, aquellos alumnos que utilizan en mayor medida estrategias motivacionales y de aprendizaje y que presentan mayores niveles de competencia percibida, obtendrán mejores calificaciones académicas.

\section{METODO}

\section{Participantes}

En la investigación participaron 478 estudiantes de tercer $(N=216,45.2 \%)$ y cuarto curso $(N=262,54.8 \%)$ de Educación Secundaria Obligatoria con edades comprendidas entre los 14 y los 18 años de edad $(M=15.68 ; S D=.95)$. La distribución conforme a la variable género fue chicos $(47.7 \%)$ y chicas $(52.3 \%)$.

\section{Instrumentos}

En relación a los estilos educativos paternos, evaluamos, mediante informe de hijos, el afecto y el control como dimensiones de la conducta paterna. Para ello se utilizaron instrumentos adaptados que este grupo ha utilizado en investigaciones previas en las que han mostrado su utilidad (Casanova, Carpio, Cerezo y García, 2004; Cerezo, De la Torre, García y Carpio, 2004; Pelegrina et al., 2002).

Escala de Aceptación. Esta escala fue diseñada basándose en la propuesta realizada por Steinberg, Lamborn, Dornbusch y Darling (1992). Evalúa el grado en que los estudiantes se sienten ayudados y comprendidos por sus padres y la medida en que éstos son sensibles a sus necesidades. La escala está compuesta por 7 ítems y su fiabilidad entendida como consistencia interna fue de $\alpha=0.68$.

Escala de Control. Siguiendo el mismo procedimiento de la escala de aceptación, se elaboró una escala de control que evaluaba el grado en que los estudiantes experimentaban supervisión por parte de sus padres y el establecimiento de límites y normas. Esta escala está compuesta por 8 ítems y su fiabilidad entendida como consistencia interna resultó ser $\alpha=0.63$.

En cuanto a la evaluación del uso que los estudiantes hacen de estrategias de aprendizaje, se utilizó una adaptación del Inventario de Estrategias de aprendizaje y Estudio LASSI de Weinstein (1987). Esta adaptación fue realizada por García (1998) para alumnos de Secundaria y consta de 63 ítems que se agrupan en diez factores de primer orden: actitud, motivación, control del tiempo, ansiedad, concentración, procesamiento de la información, selección de las ideas principales, estrategias de apoyo, autoevaluación y preparación de exámenes. Estos factores de primer orden, a su vez, se agrupan en tres factores de segundo orden: Estrategias Motivacionales de apoyo (actitud, concentración, motivación y organización del tiempo), Estrategias de aprendizaje (estrategias de apoyo, procesamiento de la información y autoevaluación) y Competencia percibida (ansiedad, preparación de exámenes y selección de ideas principales). Las estrategias motivacionales de apoyo se refieren a una serie de actividades llevadas a cabo por el alumno que puede facilitar o inhibir la utilización de determinados tipos de estrategias de aprendizaje (preparar al día los trabajos, estudiar el 
material aunque resulte aburrido, tener como meta la obtención de buenos resultados, etc.). Este factor explicaría el 47.2\% de la varianza. El factor denominado Estrategias de aprendizaje explican el $19.4 \%$ de la varianza y tiene que ver con aquellas estrategias dirigidas a la comprensión y significatividad del aprendizaje (revisar los apuntes, emplear el subrayado, seleccionar ideas principales, etc.). Por último, el factor denominado Competencia percibida explica el $7.5 \%$ de la varianza y evalúa en qué medida el alumno se considera capaz y competente para seleccionar información relevante, organizarse el tiempo de estudio, planificar el estudio, etc.

En esta investigación se han tenido en cuenta las puntuaciones obtenidas por los estudiantes en estos tres factores de segundo orden.

Además de los instrumentos anteriormente descritos, se tuvo en cuenta la información aportada por los alumnos acerca de las calificaciones obtenidas en el curso anterior en las asignaturas de lenguaje, matemáticas y ciencias sociales. Estas calificaciones fueron categorizadas en un rango de puntuaciones que va desde 1 hasta 5 (1 insuficiente... 5 sobresaliente).

\section{Procedimiento}

En primer lugar se llevó a cabo la elección de los centros educativos y se solicitó una cita con los directores para poder explicarles el objetivo del trabajo de investigación y para requerir su colaboración. Posteriormente, aquellos alumnos que voluntariamente quisieron participar en la investigación, completaron las escalas de aceptación y control y el LASSI, informando además de las calificaciones obtenidas en el curso anterior en las asignaturas de lenguaje, matemáticas y ciencias sociales.

Con las respuestas emitidas por los alumnos en las escalas de aceptación y control, se llevó a cabo la clasificación de los padres en los estilos educativos autoritario, permisivo, negligente y democrático. Para ello se siguió el procedimiento propuesto por Steinberg et al. (1992), el cual consiste en combinar las puntuaciones correspondientes al tercio superior e inferior (percentiles 33 y 66) en las dimensiones consideradas.

Mediante este procedimiento se obtuvieron tipos "puros" de padres al basarse únicamente en las puntuaciones extremas de las dimensiones de control y aceptación. Esto implica que la muestra de padres inicial se redujo considerablemente puesto que quedaron excluidos aquellos que presentaban puntuaciones intermedias en las dimensiones mencionadas.

En cuanto a las técnicas de análisis estadístico llevadas a cabo para contrastar las hipótesis planteadas, se realizaron análisis univariados de varianza con el fin de determinar la influencia de las estrategias de aprendizaje autorregulado sobre el rendimiento de los estudiantes y para conocer la posible incidencia del estilo paterno percibido por los alumnos sobre el uso de tales estrategias.

\section{RESULTADOS}

Estrategias de aprendizaje autorregulado y rendimiento académico.

En la primera hipótesis se planteaba la existencia de diferencias significativas en el rendimiento académico de los estudiantes en función de la utilización de estrategias 
de aprendizaje autorregulado. Para comprobar esta hipótesis, se formaron dos grupos de alumnos en función de las puntuaciones medias obtenidas en los tres factores de segundo orden del LASSI (puntuaciones por encima y por debajo de la media) y se comparó el rendimiento de estos dos grupos en las asignaturas de lenguaje, matemáticas y ciencias sociales mediante la realización de un análisis de varianza univariado (Tablas 1 y 2).

Tabla 1. Diferencias en el rendimiento en matemáticas, ciencias sociales y lenguaje en función de las estrategias de aprendizaje autorregulado utilizadas

\begin{tabular}{lcccccc}
\hline & \multicolumn{2}{c}{ Matemáticas } & \multicolumn{2}{c}{ Ciencias sociales } & \multicolumn{2}{c}{ Lenguaje } \\
\cline { 2 - 6 } & $F$ & $p$ & $F$ & $p$ & $F$ & 72.71 \\
Estrategias motivacionales & 48.351 & .000 & 55.86 & .000 & .000 \\
Estrategias de aprendizaje & 3.18 & .075 & 13.179 & .000 & 12.04 \\
Competencia percibida & 21.42 & .000 & 42.779 & .000 & .001 & 43.40 \\
\hline
\end{tabular}

Los datos obtenidos confirman las predicciones realizadas. En concreto, casi todas las calificaciones informadas por los alumnos son significativamente distintas dependiendo de las puntuaciones obtenidas en las tres escalas de segundo orden del LASSI (ver tabla 1). Únicamente no se encuentran tales diferencias en las calificaciones de la asignatura de matemáticas en función del uso de estrategias de aprendizaje autorregulado. En concreto, tal y como queda reflejado en la tabla 2, se puede observar que el mayor uso de estrategias incide positivamente sobre las calificaciones obtenidas en cada una de las asignaturas, de tal manera, que los estudiantes que obtuvieron mayores puntuaciones en las escalas del LASSI, obtuvieron también mejores calificaciones en el curso anterior.

Tabla 2. Medias y desviaciones típicas de las calificaciones obtenidas en matemáticas, ciencias sociales y lenguaje en función el mayor o menor uso de estrategias de aprendizaje autorregulado

\begin{tabular}{lcccc}
\hline & Grupo & Matemáticas & Ciencias sociales & Lenguaje \\
& & $M(S D)$ & $M(S D)$ & $2.50(1.18)$ \\
& 1 & $2.43(1.29)$ & $2.98(1.38)$ & $3.50(1.33)$ \\
\hline Estrategias & 2 & $3.30(1.37)$ & $3.93(1.37)$ & $2.85(1.31)$ \\
motivacionales & 1 & $2.80(1.36)$ & $3.27(1.43)$ & $3.28(1.38)$ \\
\hline Estrategias de & 2 & $3.04(1.44)$ & $3.75(1.44)$ & $2.59(1.27)$ \\
aprendizaje & 1 & $2.56(1.29)$ & $3.00(1.32)$ & $3.39(1.32)$ \\
\hline \multirow{2}{*}{ Competencia percibida } & 2 & $3.16(1.43)$ & $3.86(1.44)$ & \\
& & & &
\end{tabular}

Nota: El grupo 1 se corresponde con los alumnos que obtuvieron puntuaciones en el LASSI por debajo de la media y el grupo 2 con los que sus puntuaciones fueron superiores a la media.

Estilos educativos paternos y estrategias de aprendizaje autorregulado.

En segundo lugar y con el fin de comprobar la segunda de las hipótesis planteadas, se llevó a cabo la distribución de los padres en los cuatro estilos educativos utilizándose las dimensiones aceptación y control evaluadas mediante las escalas descritas con anterioridad.

En la tabla 3 se puede observar el número de familias incluidas dentro de cada estilo educativo paterno además de la media y desviación típica de cada grupo en las dimensiones aceptación y control. 
Tabla 3. Número de familias incluidas en cada uno de los estilos educativos paternos, puntuaciones medias y desviaciones típicas de cada una de las dimensiones evaluadas

\begin{tabular}{cccccc}
\hline & & Autoritario & Democrático & Negligente & Permisivo \\
& $N=57$ & $N=65$ & $N=67$ & $N=51$ \\
\hline \multirow{2}{*}{ Aceptación } & $M$ & 26.42 & 37.87 & 28.01 & $(2.81)$ \\
& $(S D)$ & $(4.58)$ & $(1.34)$ & 19.19 & $(1.13)$ \\
\hline \multirow{2}{*}{ Control } & $M$ & 27.40 & 26.36 & $(2.39)$ & 19.05 \\
& $(S D)$ & $(2.12)$ & $(1.36)$ & $(2.10)$ \\
\hline
\end{tabular}

Una vez clasificados los padres en función las dimensiones aceptación y control, se procedió a analizar la posible existencia de diferencias en la competencia percibida y en las estrategias motivacionales y de aprendizaje utilizadas por los alumnos, en función de los estilos educativos paternos. Para ello se llevó a cabo un análisis univariado de varianza cuyos resultados quedan reflejados en la tabla 4 , donde se puede observar la existencia de diferencias significativas en competencia percibida $\left(F_{(3,228)}=\right.$ $5.70, p<.001)$, estrategias motivacionales $\left(F_{(3,228)}=12.87, p<.000\right)$ y estrategias de aprendizaje $\left(F_{(3,228)}=11.38, p<.000\right)$.

Tabla 4. Diferencias en las estrategias de aprendizaje autorregulado encontradas en función del estilo educativo paterno

\begin{tabular}{|c|c|c|c|c|c|c|}
\hline & $\begin{array}{c}\text { Autoritario } \\
N=53\end{array}$ & $\begin{array}{c}\text { Democrático } \\
N=64\end{array}$ & $\begin{array}{c}\text { Negligente } \\
N=63\end{array}$ & $\begin{array}{c}\text { Permisivo } \\
N=48\end{array}$ & $F$ & $p$ \\
\hline $\begin{array}{l}\text { Estrategias } \\
\text { motivacionales de } \\
\text { apoyo }\end{array}$ & $\begin{array}{l}24.57 \\
(4.37)\end{array}$ & $\begin{array}{l}27.81 \\
(4.13)\end{array}$ & $\begin{array}{l}24.91 \\
(3.74)\end{array}$ & $\begin{array}{l}28.12 \\
(3.32)\end{array}$ & 12.87 & .000 \\
\hline $\begin{array}{l}\text { Estrategias de } \\
\text { aprendizaje }\end{array}$ & $\begin{array}{l}25.57 \\
(3.99)\end{array}$ & $\begin{array}{l}28.56 \\
(3.74)\end{array}$ & $\begin{array}{l}25.59 \\
(4.07)\end{array}$ & $\begin{array}{l}28.60 \\
(3.63)\end{array}$ & 11.38 & .000 \\
\hline $\begin{array}{l}\text { Competencia } \\
\text { percibida }\end{array}$ & $\begin{array}{l}23.20 \\
(3.24)\end{array}$ & $\begin{array}{l}24.20 \\
(1.89)\end{array}$ & $\begin{array}{l}22.81 \\
(2.72)\end{array}$ & $\begin{array}{l}24.54 \\
(2.22)\end{array}$ & 5.70 & .001 \\
\hline
\end{tabular}

Tabla 5. Diferencias encontradas en la utilización de estrategias de aprendizaje en función del tipo de estilo educativo paterno

\begin{tabular}{|c|c|c|c|c|}
\hline & & & $D M S$ & $p$ \\
\hline \multirow{6}{*}{$\begin{array}{l}\text { Estrategias } \\
\text { motivacionales de } \\
\text { apoyo }\end{array}$} & \multirow{3}{*}{ Autoritario } & Democrático & $-3.242 *$ & .000 \\
\hline & & Negligente & -.338 & .642 \\
\hline & & Permisivo & $-3.545^{*}$ & .000 \\
\hline & \multirow{2}{*}{ Democrático } & Negligente & $2.903^{*}$ & .000 \\
\hline & & Permisivo & -.303 & .683 \\
\hline & Negligente & Permisivo & $-3.207 *$ & .000 \\
\hline \multirow{6}{*}{$\begin{array}{l}\text { Estrategias de } \\
\text { aprendizaje }\end{array}$} & \multirow{3}{*}{ Autoritario } & Democrático & $-2.995 *$ & .000 \\
\hline & & Negligente & -.0202 & .978 \\
\hline & & Permisivo & $-3.031 *$ & .000 \\
\hline & \multirow{2}{*}{ Democrático } & Negligente & $2.975^{*}$ & .000 \\
\hline & & Permisivo & -.0364 & .961 \\
\hline & Negligente & Permisivo & $-3.011 *$ & .000 \\
\hline \multirow{6}{*}{ Competencia percibida } & \multirow{3}{*}{ Autoritario } & Democrático & $-.999 *$ & .037 \\
\hline & & Negligente & .386 & .419 \\
\hline & & Permisivo & $-1.340 *$ & .009 \\
\hline & \multirow{2}{*}{ Democrático } & Negligente & $1.385^{*}$ & .003 \\
\hline & & Permisivo & -.3411 & .486 \\
\hline & Negligente & Permisivo & $-1.726^{*}$ & .001 \\
\hline
\end{tabular}

Con el fin de descubrir entre qué tipos de padres se producían diferencias significativas con respecto a las estrategias utilizadas por los alumnos, se llevaron a cabo 
comparaciones post hoc (comparación múltiple por parejas de la diferencia menos significativa), mediante las cuales se encontró que los alumnos que describían a sus padres como indiferentes y autoritarios diferían significativamente de los estudiantes cuyos padres fueron clasificados como democráticos y permisivos, no encontrándose diferencias significativas entre hijos de padres democráticos y permisivos ni entre hijos de padres autoritarios e indiferentes (tabla 5). En concreto y tal y como queda reflejado en la tabla 4, los hijos que percibían a sus padres como democráticos y como permisivos presentan mayores puntuaciones medias en cada una de las estrategias de aprendizaje consideradas en este estudio.

\section{DISCUSIÓN}

La hipótesis central de esta investigación plantea que los estilos educativos paternos ejercen una influencia indirecta sobre el rendimiento de los estudiantes actuando a través de las estrategias de aprendizaje autorregulado utilizadas por éstos. Los resultados obtenidos permiten corroborar la existencia de tales influencias.

$\mathrm{Al}$ igual que se ha encontrado en numerosas investigaciones (De la Fuente, 2004; Torrano y González-Torres, 2004; Valle, Cabanach, Rodríguez, Núñez y González-Pienda, 2005), en este trabajo los datos apoyan el supuesto de que el logro académico de los alumnos se incrementa en la medida en que éstos utilizan mayor cantidad de estrategias de aprendizaje y se comportan de modo autorregulado. Además, también se ha comprobado que el estilo educativo paterno incide significativamente sobre dichas estrategias.

En consonancia con los resultados obtenidos por Erden y Uredi (2008), hemos encontrado que en general, los alumnos cuyos progenitores fueron clasificados como democráticos y permisivos presentan en mayor medida estrategias motivacionales y de aprendizaje, si los comparamos con los adolescentes cuyos padres son descritos como autoritarios e indulgentes. En cuanto a la capacidad percibida, también se ha demostrado que el estilo democrático es más beneficioso, de tal manera que los estudiantes cuyos padres habían sido clasificados como democráticos y permisivos, se consideraban a sí mismos como más competentes en el ámbito cognitivo que los hijos de padres autoritarios y negligentes.

Los padres democráticos y permisivos se caracterizan por presentar altos niveles de aceptación e implicación. Estos padres fomentan la autorregulación de sus hijos, atendiendo también a sus necesidades y demandas (Baumrind, 1991). Si revisamos la investigación existente sobre este tema, encontramos la existencia una relación positiva entre las actitudes de apoyo manifestadas por los padres y las conductas de autorregulación, el uso de estrategias de aprendizaje autorregulado y el rendimiento académico de los alumnos (Grolnick y Ryan, 1989; Otto, Perels y Schmitz, 2008).

Sin embargo, los padres autoritarios y los negligentes no manifiestan tanta aceptación, no suelen atender a las necesidades y demandas de sus hijos, fomentando así falta de confianza en sí mismos y un deficiente uso de habilidades de aprendizaje autorregulado (Erden y Uredi, 2008). 
De acuerdo con estos resultados se puede concluir que la aceptación, entendida como la ayuda y comprensión de los padres, es importante en la determinación del aprendizaje y el logro académico de los adolescentes, ya que les ayuda a llevar a cabo aprendizajes autorregulados. Sin embargo, no se ha podido demostrar la influencia del control sobre la puesta en marcha de estrategias de aprendizaje autorregulado. Puede que esta dimensión del estilo educativo paterno esté relacionada con otros aspectos implicados en el aprendizaje académico, como son las metas perseguidas por los estudiantes o las atribuciones causales realizadas por éstos ante los resultados académicos. Por este motivo, en posteriores investigaciones sería interesante abordar el estudio de la relación existente entre los estilos educativos de los padres y estas otras variables implicadas en el aprendizaje escolar.

También, en el futuro sería interesante considerar otras dimensiones del estilo educativo paterno como es el grado de implicación y la presencia de diversos comportamientos en los padres, como las conductas de incitación parental a la autorregulación, los cuales ayudan a los adolescentes a desarrollar ciertas habilidades autorregulatorias (González-Pienda et al., 2002; Martínez-Pons, 1996).

\section{REFERENCIAS}

Balzano, S. (2002). Las construcciones culturales sobre el éxito y el fracaso escolar y sus implicaciones sobre los modelos educativos en Argentina. Cultura y Educación, 3(14), 283-296.

Ballantine, J. (2001). Raising competent kids: The authoritative parenting style. Childhood Education, 78, 46-47.

Baumrind, D. (1991). Parenting styles and adolescent development. En R.M. Lerner, A.C. Peterson y J. Brooks-Gunn (Eds.) Encyclopedia of adolescence (pp. 746-758). New York: Garland Publishing, INC.

Burchinal, M.R., Peisner-Feinberg, E., Pianta, R. y Howes, C. (2002). Development of academic skills from preschool through second grade: Family and classroom predictors of developmental trajectories. Journal of School Psychology, 40, 415-436.

Casanova, P.F.; Carpio, M.V.; Cerezo, M.T. y García, M.C. (2004). Comparación de dos procedimientos de clasificación de estilos de padres en alumnos de Enseñanza Secundaria Obligatoria. En F. Miras, N. Yuste y F. Valls (Eds.), Calidad Educativa. Almería, Universidad de Almería.

Cerezo, M.T., De la Torre, M.J., García, M.C. y Carpio, M.V. (2004). Influencia de los estilos educativos de los padres sobre la motivación académica. En F. Miras y N. Yuste (Comps.), Calidad Educativa. Almería: Servicio de Publicaciones de la Universidad de Almería.

Coloma, J. (1993). Estilos educativos paternos. En J.Mª Quintana Cabanas (Coord.): Pedagogía familiar (pp. 45-58). Madrid: Narcea.

De la Fuente, J. (2004). Perspectivas recientes en el estudio de la motivación: la teoría de la orientación a meta. Revista Electrónica de Investigación Psicoeducativa, 2(1), 35-62.

Dornbusch, S.M., Ritter, P.L., Leiderman, P.H., Roberts, D.F. y Fraleigh, M.J. (1987). The relation of parenting styles to adolescent school performance. Child Development, 58, 1244-1257.

Erden, M. y Uredi, I. (2008). The effect of perceived parenting styles on self-regulated learning strategies and motivational beliefs. International Journal about Parents in Education, 2(1), 25-34. 
García, M. (1998). Estrategias de aprendizaje en la Educación Secundaria Obligatoria. Tesis Doctoral. Departamento de Psicología. Universidad de Oviedo.

Glasgow, K.L. Dornbusch, S.M. Troyer, L. Steinberg, L. y Ritter, P.L. (1997) Parenting styles, adolescent's attribution and educational outcomes in nine heterogeneous high schools, Child Development, 68(3), 507-529.

González, A.R., Holbein, M.F. y Quilter, S. (2002). High school student's goal orientation and their relationship to perceived parenting styles. Contemporary Educational Psychology, 27, 450-470.

González-Pienda, J.A., Núñez, J.C., González-Pumariega, S., Álvarez, L., Roces, C. y García, M. (2002). A structural equation model of parental involvement, motivational and aptitudinal charateristics and academia achievement. The Journal of Experimental Education, 70(3), 257-287.

González-Pienda, J.A., Núñez, J.C, Álvarez, L., González-Pumariega, S., Roces, C., González, P., Muñiz, R. y Bernardo, A. (2002). Inducción parental a la autorregulación, autoconcepto y rendimiento académico. Psicothema, 14(4), 853-860.

Grolnick, W.S. y Ryan, R.M. (1989). Parent styles associated with children's self-regulation and competence in school. Journal of Educational Psychology, 81, 143-154.

Hickman, G.P., Bartholomae, S. y McKenry, P.C. (2000). Influence of parenting styles on the adjustment and academic achievement of traditional college freshman. Journal of College Student Development, 41, 41-52.

Lamborn, S.D., Mounts, N., Steinberg, L. y Dornbusch, S.M. (1991). Patterns of competence and adjustment among adolescents from authoritative, authoritarian, indulgent and neglectful families. Child Development, 62, 1049-1065.

Maccoby, E.E. y Martin, J.A. (1983). Socialization in the context of the family. Parent-child interaction. En E.M. Hetherington (Ed.), Handbook of child psychology. Vol. 4 Socialization, personality and social development (pp. 1-101). New York: Wiley.

Martínez-Pons, M. (1996). Test of a model of parental inducement of academic self-regulation. The Journal of Experimental Education, 64, 213-227.

Otto, B., Perels, F. y Schmitz, B. (2008). The correlation of parental behavior with the selfregulative learning of elementary school students. Psychologie in Erziehung und Unterricht, 55(4), 288-300.

Pelegrina, S., García, M.C. y Casanova, P. (2002). Los estilos educativos de los padres y la competencia académica de los adolescentes. Infancia y Aprendizaje 25(2), 147-168.

Peralbo, M. y Fernández, M.L. (2003). Estructura familiar y rendimiento escolar en Educación Secundaria Obligatoria. Revista Galego-Portuguesa de Psicoloxía e Educación, 7(8), 309322.

Pérez De Pablos, S. (2003) El papel de los padres en el éxito escolar de los hijos. Madrid, Santillana.

Pintrich, P.R. (1999). The role of motivation in promoting and sustaining self-regulated learning. International Journal of Educational Research, 31, 459-470.

Schunk, D.H. y Zimmerman, B.J. (2008). Motivating and self-regulated learning. New York: Lawrence Erlbaum Associates.

Steinberg, L., Elmen, J.D. y Mounts, N.S. (1989). Authoritative parenting, psychosocial maturity, and academic success among adolescents. Child Development, 60, 1424-1436.

Steinberg, L., Lamborn, S.D., Dornbusch, S.M. y Darling, N. (1992). Impact of parenting practices on adolescent achievement: authoritative parenting, school involvement and encouragement to succeed. Child Development, 63, 1266-1281.

Torrano, F. y González-Torres, M.C. (2004). El aprendizaje autorregulado: presente y futuro de la investigación. Revista Electrónica de Investigación Psicoeducativa, 2(1), 1-34. 
Torío, S., Peña, J.V. e Inda, M. (2008). Estilos de educación familiar. Psicothema, 2(1), 62-70.

Torío, S., Peña, J.V. y Rodríguez, M.C. (2008). Estilos educativos parentales: revisión bibliográfica y reformulación teórica. Teoría de la Educación, 20, 151-178.

Valle, A., Rodríguez, S., Cabanach, R.G., Núñez, J.C. y González-Pienda, J.A. (2005). Self-Worth protection strategies in higher educational students: Exploring a model of predictors and consequences. En R. Nata (Ed.), News directions in higher education (pp. 99-126). New York: Nova Science Publishers.

Weinstein, C.E (1987). LASSI (Learning and Study Strategies Inventory). Clearwater, F.L.H y H Publishing Company.

Recibido: 3 de marzo de 2010

Recibido modificaciones: 11 de mayo de 2010

Aceptado: 14 de junio de 2010 